\title{
Gas-exchange parameters and morphological features of festulolium (Festulolium braunii K. Richert A. Camus) in response to nitrogen dosage
}

\author{
G. MASTALERCZUK* , B. BORAWSKA-JARMUŁOWICZ* , H. M. KALAJI*,\$, ${ }^{*}$, P. DĄBROWSKI ${ }^{* * *}$, \\ and J. PADEREWSKI ${ }^{\#}$
}

Department of Agronomy, Faculty of Agriculture and Biology", Department of Plant Physiology, Faculty of Agriculture and Biology**, Department of Environmental Improvement, Faculty of Civil and Environmental Engineering ${ }^{* * *}$, Department of Experimental Design and Bioinformatics, Faculty of Agriculture and Biology", Warsaw University of Life Sciences WULS-SGGW, Nowoursynowska 159, 02-776 Warsaw, Poland

SI Technology, Górczewska 226C/26, 01-460 Warsaw, Poland ${ }^{\S}$

\begin{abstract}
The response of some photosynthetic parameters $\left(\mathrm{CO}_{2}\right.$ assimilation, transpiration rate, stomatal conductance, intercellular $\mathrm{CO}_{2}$ concentration, water-use efficiency, and chlorophyll content), shoot development, and the morphological features of the root system to differentiated conditions of nitrogen supply was tested in festulolium (Festulolium braunii K. Richert A. Camus) varieties (Felopa and Sulino). Nitrogen fertilization with no nitrogen added $[0 \mathrm{~g}(\mathrm{~N})]$, single dosage $[0.23 \mathrm{~g}(\mathrm{~N})]$, and double dosage $[0.46 \mathrm{~g}(\mathrm{~N})]$ per pot and per year was applied. Lack of nitrogen resulted in formation of longer and finer roots and lowered chlorophyll content, $\mathrm{CO}_{2}$ assimilation, and water-use efficiency, resulting in lower dry matter accumulation. Application of both dosages of nitrogen resulted in improved aboveground features, while root features were enhanced without nitrogen fertilization. Dependence between physiological parameters and morphological traits was significant and positively correlated in the case of the aboveground parts of plants and negatively correlated to the belowground parts.
\end{abstract}

Additional key words: $\mathrm{CO}_{2}$ assimilation; nitrogen; root diameter; specific root length.

\section{Introduction}

The productivity and botanical composition, especially of temporary grassland, are influenced markedly by nitrogen and water availability. Festulolium (Lolium $\times$ Festuca) hybrids are more frequently used as forage grasses because of their great ability to combine characteristics of their parent species (Boller et al. 2010, Østrem et al. 2013). Varieties of festulolium belong to grasses with a high potential for yield, especially in the first year, at high doses of nitrogen (Gutmane and Adamovics 2009). They give higher yields by about $20 \%$ in comparison to other forage grass species (e.g. Festuca pratensis) (Kryszak et al. 2002). For this reason, they are used in intensive feed production and sown on the arable land of temporary grasslands, in both pure strains and in mixtures (Nekrošas and Kemešytė 2007, Østrem and Larsen 2008).

The investigation of crop physiological function in order to raise the yield is always an important issue in agronomy, plant breeding, and ecology. Photosynthesis is the key process of plant metabolism which is strongly influenced by environmental conditions. Nutrients availability affects significantly the photochemical part of photosynthesis (Kalaji et al. 2014). The effects of nitrogen accessibility on physiological processes of plants have been well documented in numerous studies (Olszewska 2008a, Živčák et al. 2014). Nitrogen is also known to play an important role in coordination between photosynthesis process and supplying plant organ acceptors with photoassimilates (Starck 2010).

The utilization of water and soil nutrients by a plant is dependent upon a size of its root system, which plays an essential role in physiological processes. Typically, if the roots are more developed, the plants use minerals more effectively during the growing season. The root biomass of meadow and pasture plants is accumulated in large amounts, particularly on temporary grassland (Stypiński and Mastalerczuk 2005, Soussana et al. 2014) and

Received 18 April 2016, accepted 7 September 2016, published as online-first 17 October 2016.

${ }^{+}$Corresponding author; phone: + 48 664943484, e-mail: hazem@kalaji.pl,

Abbreviations: $\mathrm{B}$ - total dry biomass of plants; $\mathrm{Chl}$ - chlorophyll; $C_{\mathrm{i}}$ - intercellular $\mathrm{CO}_{2}$ concentration; $\mathrm{D}$ - average diameter of roots; DAS - days after sowing; DD - double dose; $E$ - transpiration rate; $g_{\mathrm{s}}$ - stomatal conductance; LN - leaf number; $P_{\mathrm{N}}$ - net photosynthetic rate; RDM - root dry mass; RL - total length of roots; R/S - root/shoot ratio; SA - surface area of roots; SD - single dose; SDM - shoot dry mass; SN - shoot number; SPAD - leaf greenness index; SRL - specific root length; WUE - water-use efficiency. 
therefore it is an important source of organic matter in soil (Fiala 2010). The root length and diameter of distribution are major characteristics of a plant's root system. This is crucial since a root's function may vary depending on its diameter (Hodge et al. 2009). Extended root length is an important feature for the acquisition of nutrients, especially for nitrogen, when its availability is scarce. In such conditions, plants allocate proportionally more of their biomass to the roots, which are finer (Ryser and Lambers 1995). The most often investigated root property, next to root diameter, is its length per unit of dry mass of the root system (specific root length, SRL) (Ryser 2006, Picon-Cochard et al. 2012). The higher this indicator is, the better is the root branching, which allows plants to survive periods of nutrient and water deficiency (Hill et al. 2006). Nevertheless, the intraspecific variability among traits is often neglected, especially in the case of root traits, which are less well studied than leaf traits (Kattge et al. 2011). Several studies have found differences in the

\section{Materials and methods}

Plant material and growth conditions: The experiment was carried out in a greenhouse at the Warsaw University of Life Sciences-SGGW in Poland in the years 2012 and 2013, between April and October. The study was performed on plants of two Polish forage varieties of festulolium (Festulolium braunii, K. Richert and A. Camus) - Felopa (F) and Sulino (S). In each year, seeds were sown in three replications. Fifteen days after sowing, the poorly developed seedlings were removed and investigations were performed on four plants per pot. Plants were cut five times during the study period [at 40 , $80,105,135$, and 180 days after sowing (DAS)]. The pots (18 $\mathrm{cm}$ in diameter and $15 \mathrm{~cm}$ in height) were filled by podzolic soil formed from loamy sand. Soil water regime was controlled every two days by weighing each pot and restoring soil water content to $70 \%$ capillary water capacity. The average soil chemical characteristics were as follows: $9.5 \mathrm{~g}$ (total organic C) $\mathrm{kg}^{-1}, 1.01 \mathrm{~g}(\mathrm{~N}) \mathrm{kg}^{-1}, 94.5$ $\mathrm{mg}$ (available $\mathrm{P}) \mathrm{kg}^{-1}, 99.6 \mathrm{mg}(\mathrm{K}) \mathrm{kg}^{-1}$, and $40.0 \mathrm{mg}(\mathrm{Mg})$ $\mathrm{kg}^{-1}$. The soil $\mathrm{pH}_{\mathrm{KCl}}$ was 5.2. Mineral fertilizers were applied at the following rates (per pot per year): $0.15 \mathrm{~g} \mathrm{P}$ (triple superphosphate), $0.25 \mathrm{~g} \mathrm{~K}$ (potassium chloride). Differentiated nitrogen $(\mathrm{N})$ fertilization was supplied as ammonium nitrate in doses ( $\mathrm{g}$ per pot per year): no nitrogen (0), 0.23 - single dose (SD), 0.46 - double dose (DD) at five equal rates during plant growth (before sowing and after each cut). Nitrogen fertilization was applied at a dosage equivalent to 90 and $180 \mathrm{~kg}(\mathrm{~N}) \mathrm{ha}^{-1}$ per year (SD and DD, respectively).

Conditions in the greenhouse during the study period, i.e. humidity of air day/night [\%], temperature day/night $\left[{ }^{\circ} \mathrm{C}\right]$, and radiation for $24 \mathrm{~h}\left[\mathrm{~W} \mathrm{~m}^{-2}\right]$ were automatically controlled by opening and closing the windows and curtains and registered by the climate control computer system (HortiMaX B.V., the Netherlands). Temperature development of root mass under nitrogen fertilization (James 2008, Gastal et al. 2010).

The amount of nitrogen applied to plants should be optimal for both shoot and root development as well as for the relations between them. There is a lack of multiparametric studies on the effect of important nutrient element supporting high photosynthetic rate and formation of above (shoot) as well as below (root) biomass of grasses. Therefore, the study of a plant's root and shoot biomass allocation as an integrated response to environmental factors as related to nitrogen fertilization is still underway. The aim of this work was to study the effect of nitrogen availability in the soil on photosynthesis process and to find out how the latter can modify the root system of festulolium plants. Therefore, we investigated the changes in gas-exchange parameters, physiological features, and the morphological characteristics of the root system and shoot development of festulolium varieties, as a response to different dosages of nitrogen.

distribution was favourable for plants during both growing periods, with the spring temperature being especially moderate (April $18 / 15.5^{\circ} \mathrm{C}$ and $16 / 14^{\circ} \mathrm{C}$, May $25 / 18^{\circ} \mathrm{C}$ and $23 / 17^{\circ} \mathrm{C}$, respectively in 2012 and 2013). The highest air temperature for both years occurred in July (about $29^{\circ} \mathrm{C}$ during the day). The air humidity values in the greenhouse fluctuated from about $50-55 \%$ during the day to about $40-45 \%$ at night. Irradiation varied from about $150 \mathrm{~W} \mathrm{~m}^{-2}$ in the spring to $240 \mathrm{~W} \mathrm{~m}^{-2}$ during the summer months, and then gradually decreased to about $100 \mathrm{~W} \mathrm{~m}^{-2}$ in October.

Methods and measurements: A portable photosynthesis measuring system (LCpro+, ADC BioScientific Ltd., England) was used to measure $\mathrm{CO}_{2}$ assimilation $\left(P_{\mathrm{N}}\right)$ $\left[\mu \mathrm{mol}\left(\mathrm{CO}_{2}\right) \mathrm{m}^{-2} \mathrm{~s}^{-1}\right]$, transpiration rate $(E)\left[\mathrm{mmol}\left(\mathrm{H}_{2} \mathrm{O}\right)\right.$ $\left.\mathrm{m}^{-2} \mathrm{~s}^{-1}\right]$, stomatal conductance $\left(g_{\mathrm{s}}\right)\left[\mathrm{mol}\left(\mathrm{H}_{2} \mathrm{O}\right) \mathrm{m}^{-2} \mathrm{~s}^{-1}\right]$, and intercellular $\mathrm{CO}_{2}$ concentration $\left(C_{\mathrm{i}}\right)\left[\mu \mathrm{mol}\left(\mathrm{CO}_{2}\right) \mathrm{mol}^{-1}\right]$. Measurements were performed at the ambient $\mathrm{CO}_{2}(\mu \mathrm{mol}$ $\left.\mathrm{m}^{-2} \mathrm{~s}^{-1}\right)$ concentration and saturating irradiance $[1,000$ $\mu$ mol(photon) $\mathrm{m}^{-2} \mathrm{~s}^{-1}$ ] from 09:00 to 11:00 h. The leaf greenness index (SPAD) was determined by using a SPAD-502 optical chlorophyll meter (Minolta, Japan). Photosynthetic water-use efficiency (WUE) was evaluated as a ratio: $\mathrm{WUE}=P_{\mathrm{N}}\left[\mu \operatorname{mol}\left(\mathrm{CO}_{2}\right) \mathrm{m}^{-2} \mathrm{~s}^{-1}\right] / E\left[\mathrm{mmol}\left(\mathrm{H}_{2} \mathrm{O}\right)\right.$ $\mathrm{m}^{-2} \mathrm{~s}^{-1}$.

Physiological parameters and SPAD values were measured in the tillering stage of plants on the youngest three fully developed leaves of each plant per pot after 40, 80, 105,135 , and 180 DAS.

The morphometric measurements of plants were performed three times: at the beginning (40 DAS) and completion of the tillering stage ( 80 and 180 DAS) on each plant per pot (four plants) of each variant repeated three times $(n=36)$. The shoot (SN) and leaf (LN) numbers per plant were evaluated. To determine the above- and below- 
ground biomass, roots were cut from shoots at tillering nodes. In order to separate the roots of plants from the soil, pot content was washed in a sieve with a mesh size of $1-0.3 \mathrm{~mm}$ in moderate stream of water and cleaned of soil parts. The collected root material was scanned with an Epson Perfection V700 Photo optical scanner (resolution 400 pdi) and converted to morphometric analysis using software WinRhizo 2012 (Regent Instruments Inc., Canada). The total length (RL) [cm per plant], the average diameter (D) $[\mathrm{mm}]$ and the surface area $(\mathrm{SA})\left[\mathrm{cm}^{2}\right]$ of roots were measured. In order to estimate root distribution, roots were sorted into six size classes according to their diameter $(<0.5,0.5-1,1-1.5,1.5-2,2-2.5$, and $2.5-3 \mathrm{~mm})$. Plant samples were then dried at $70^{\circ} \mathrm{C}$ to constant mass and the total dry biomass of plants (B) [g per plant], the shoot dry mass (SDM) [g per plant] and root dry mass (RDM) [g per plant] was determined. From this primary data the root/shoot ratio $(\mathrm{R} / \mathrm{S})\left[\mathrm{g} \mathrm{g}^{-1}\right]$ and specific root length (SRL) $\left[\mathrm{m} \mathrm{g}^{-1}\right]$ were derived:

$\mathrm{R} / \mathrm{S}\left[\mathrm{g} \mathrm{g}^{-1}\right]=$ roots dry mass/shoots dry mass

$\mathrm{SRL}\left[\mathrm{m} \mathrm{g}^{-1}\right]=$ root length/dry mass

Statistical analysis: The data presented are the mean values from the years 2012-2013, as a result of a similar reaction of the plants examined to the applied amounts of nitrogen uncovered during both study years. Values obtained were of plant features for two varieties of festulolium, under three doses of nitrogen, examined in three (for morphological features) or five (for gasexchange parameters) terms of observation, and were arranged in a four-way classification data set: variety-byplant, feature-by-observation, time-by-nitrogen dose. The two-way analysis of variance ( $A N O V A)$ was done for each single combination of feature and term of observation in

\section{Results}

The response of festulolium physiological parameters to the $\mathrm{N}$ application was differentiated during the study period (Tables 1, 2). Almost all parameters, except the $E$, had the lowest values at the beginning of the tillering phase (40 DAS). The values of the leaf greenness index (SPAD) in plants increased when the applied $\mathrm{N}$ dosage was higher, irrespective of the term of measurement. Substantially lower SPAD values were recorded in the Sulino variety. $\mathrm{CO}_{2}$ assimilation $\left(P_{\mathrm{N}}\right)$ was dependent on the $\mathrm{N}$ application. It was noted that $P_{\mathrm{N}}$ values were significantly lower in the absence of the $\mathrm{N}$ dosage compared to fertilized objects. Simultaneously, there was no difference in $P_{\mathrm{N}}$ between a $\mathrm{SD}$ and DD of nitrogen. The values of $P_{\mathrm{N}}$ also changed in the following terms of observation and were the highest at 80 and 180 DAS. Among the tested varieties of festulolium, Sulino was characterized by its significantly higher values of $P_{\mathrm{N}}$ than that of Felopa (by 7.5\%). E per unit of leaf area in the initial period of experiment (40 and 80 DAS) was significantly higher with SD of nitrogen applied to the plants of the Felopa variety. However, in the order to check the influence of $\mathrm{N}$ dose on plant morphological and physiological features. The factors were: $\mathrm{N}$ dose (3 levels) and variety (2 levels). Tukey's HSD test was made for every significant factor $(p<0.05)$. Relations between some selected morphological traits and physiological parameters were assessed by a correlation analysis. The collected data was standardized for each combination of feature-by-term separately in order to relatively compare the values of plant features at different terms. The main effects of combination term-by-feature were omitted because: (1) the objective of the study was not to demonstrate that the plants were growing in time (the values of plant features, such as SDM, are bigger at the end) and (2) the plant features were described with different units. The standardized data of morphological features was analyzed with a principle component analysis (PCA) and presented as a bi-plot graph. The first factor of PCA was the combination of plant features and varieties, whereas the second factor was the combination of terms of observation and nitrogen doses. The roots of plants were recorded for their length with a defined class of diameter. The two-way $A N O V A$ was done to check if the length of roots depended on the nitrogen dose for studied varieties. The factors were: $\mathrm{N}$ dosage (three levels) and variety (two levels), with the dependent variable being the root length with a defined class of diameter. The analyses took into account each single combination of term and class of diameter separately and the Tukey's HSD test was made for every significant factor $(p<0.05)$. All computations were performed with the use of the $R$ language (R, 2013). Applied procedures: $l m$ (for features of linear models), prcomp (biplot calculations), ANOVA from package car (Fox and Weisberg 2011), and Tukey's HSD test from package agricolae (Mendiburu 2012).

latter period (135 DAS), this variety showed lower values of $E$ in the absence of fertilization. $C_{\mathrm{i}}$ was affected by the varieties of festulolium, especially at the end of the study (135 and 180 DAS). The Felopa variety was characterized by higher values of $C_{\mathrm{i}}$ than that of the Sulino variety (by $7.5 \%$ and $3.3 \%$, respectively). The nitrogen application was influential for $g_{\mathrm{s}}$, especially at 80 and 135 DAS. A significant interaction between plant varieties and nitrogen dosage was noted as well. The use of nitrogen increased the values of this parameter, especially in Felopa variety plants (Tables 1,2).

The WUE was significantly affected by the $\mathrm{N}$ dose. The highest WUE values were recorded in plants fertilized with a DD of N. The varieties of festulolium tested differed significantly with respect to the plant WUE as well, but only in two terms of observations (40 and 80 DAS). The Sulino variety showed higher values of WUE by about $20 \%$, as compared to the Felopa variety (Tables 1,2 ).

The analysis of ANOVA showed that some of festulolium morphological plant features were significantly 
Table 1. Two-way ANOVA analysis showing significance of obtained results of leaf greenness index (SPAD), net photosynthetic rate $\left(P_{\mathrm{N}}\right)$, transpiration rate $(E)$, intercellular $\mathrm{CO}_{2}$ concentration $\left(C_{\mathrm{i}}\right)$, stomatal conductance $\left(g_{\mathrm{s}}\right)$, and water-use efficiency (WUE) relevant to the variety of festulolium (Felopa, Sulino) and nitrogen dose (N0 - no nitrogen, N1 - single, and N2 - double dose) in terms of observation $\left(40,80,105,135\right.$, and 180 days after sowing). ${ }^{*}, * * * * *$ - significance at the $0.05,0.01$, and 0.001 probability levels, respectively, ns - not significant.

\begin{tabular}{|c|c|c|c|c|c|c|c|}
\hline Factors & $\begin{array}{l}\text { Term } \\
\text { [DAS] }\end{array}$ & $\begin{array}{l}\text { Parameter } \\
\text { SPAD index }\end{array}$ & $P_{\mathrm{N}}$ & $E$ & $C_{\mathrm{i}}$ & $g_{\mathrm{s}}$ & WUE \\
\hline Variety & \multirow[t]{3}{*}{40} & ns & $* * *$ & ns & ns & ns & $* *$ \\
\hline $\mathrm{N}$ dose & & $* * *$ & $* * *$ & $* *$ & ns & ns & $*$ \\
\hline Variety $\times \mathrm{N}$ dose & & $* * *$ & $*$ & $*$ & ns & ns & $* * *$ \\
\hline Variety & \multirow[t]{3}{*}{80} & $* *$ & $* *$ & ns & ns & $*$ & $*$ \\
\hline $\mathrm{N}$ dose & & $* * *$ & $* * *$ & ns & ns & $* *$ & $* * *$ \\
\hline Variety $\times \mathrm{N}$ dose & & $* *$ & $* * *$ & $*$ & ns & $* *$ & $* *$ \\
\hline Variety & \multirow[t]{3}{*}{105} & $* * *$ & ns & ns & ns & ns & ns \\
\hline $\mathrm{N}$ dose & & $* *$ & $* * *$ & ns & ns & ns & $* * *$ \\
\hline Variety $\times \mathrm{N}$ dose & & * & $*$ & ns & ns & ns & $*$ \\
\hline Variety & \multirow[t]{3}{*}{135} & $* *$ & $* * *$ & $* *$ & $* * *$ & ns & ns \\
\hline $\mathrm{N}$ dose & & $* * *$ & $* * *$ & $* *$ & $* *$ & $*$ & $* * *$ \\
\hline Variety $\times \mathrm{N}$ dose & & $* * *$ & $* * *$ & $*$ & $*$ & $*$ & $* * *$ \\
\hline Variety & \multirow[t]{3}{*}{180} & $* *$ & ns & ns & * & ns & ns \\
\hline $\mathrm{N}$ dose & & $* * *$ & $* * *$ & ns & ns & ns & $* * *$ \\
\hline Variety $\times \mathrm{N}$ dose & & $* *$ & $*$ & ns & ns & $*$ & $*$ \\
\hline
\end{tabular}

Table 2. Effect of nitrogen application (N0 - no nitrogen, N1 - single, and N2 - double dose) on leaf greenness index (SPAD), net photosynthetic rate $\left(P_{\mathrm{N}}\right)$, transpiration rate $(E)$, intercellular $\mathrm{CO}_{2}$ concentration $\left(C_{\mathrm{i}}\right)$, stomatal conductance $\left(g_{\mathrm{s}}\right)$, and water-use efficiency (WUE) of festulolium varieties (Felopa, Sulino) in terms of observation (40, 80, 105, 135, and 180 days after sowing, DAS). Mean values in each term of observation (within varieties) followed by the same letter are not significantly different $(p<0.05)$ by the Tukey's HSD test.

\begin{tabular}{|c|c|c|c|c|c|c|c|c|c|c|c|}
\hline \multirow[t]{2}{*}{ Parameter } & \multicolumn{3}{|c|}{$\mathrm{N}$ dose $40 \mathrm{DAS}$} & \multicolumn{2}{|l|}{80 DAS } & \multicolumn{2}{|c|}{105 DAS } & \multicolumn{2}{|c|}{$135 \mathrm{DAS}$} & \multicolumn{2}{|l|}{$180 \mathrm{DAS}$} \\
\hline & & Felopa & Sulino & Felopa & Sulino & Felopa & Sulino & Felopa & Sulino & Felopa & Sulino \\
\hline \multirow[t]{3}{*}{ SPAD [SPAD unit] } & N0 & $29.61^{\mathrm{b}}$ & $25.40^{\mathrm{a}}$ & $39.17^{\mathrm{ab}}$ & $35.93^{\mathrm{a}}$ & $39.88^{\mathrm{ab}}$ & $38.13^{\mathrm{a}}$ & $32.29^{\mathrm{b}}$ & $26.61^{\mathrm{a}}$ & $27.73^{\mathrm{a}}$ & $24.98^{\mathrm{a}}$ \\
\hline & N1 & $35.72^{c}$ & $35.62^{c}$ & $43.49^{\mathrm{ab}}$ & $41.40^{\mathrm{ab}}$ & $43.46^{\mathrm{bc}}$ & $41.18^{\mathrm{ab}}$ & $40.59^{c}$ & $40.49^{c}$ & $36.70^{\mathrm{bc}}$ & $34.90^{\mathrm{b}}$ \\
\hline & $\mathrm{N} 2$ & $37.54^{\mathrm{cd}}$ & $38.45^{\mathrm{d}}$ & $49.67^{\mathrm{c}}$ & $42.67^{\mathrm{ab}}$ & $48.73^{\mathrm{d}}$ & $45.37^{\mathrm{cd}}$ & $46.38^{\mathrm{d}}$ & $42.99^{\mathrm{cd}}$ & $38.18^{c}$ & $37.21^{\mathrm{b}}$ \\
\hline \multirow[t]{3}{*}{$P_{\mathrm{N}}\left[\mu \mathrm{mol}\left(\mathrm{CO}_{2}\right) \mathrm{m}^{-2} \mathrm{~s}^{-1}\right]$} & N0 & $4.30^{\mathrm{a}}$ & $5.03^{\mathrm{b}}$ & $8.21^{\mathrm{a}}$ & $8.16^{\mathrm{a}}$ & $7.22^{\mathrm{ab}}$ & $6.11^{\mathrm{a}}$ & $4.17^{\mathrm{a}}$ & $5.92^{\mathrm{b}}$ & $8.38^{\mathrm{a}}$ & $8.06^{\mathrm{a}}$ \\
\hline & $\mathrm{N} 1$ & $5.00^{\mathrm{b}}$ & $6.42^{\mathrm{c}}$ & $11.04^{\mathrm{b}}$ & $12.77^{\mathrm{bc}}$ & $8.66^{\mathrm{bc}}$ & $9.10^{\mathrm{c}}$ & $9.27^{\mathrm{c}}$ & $10.41^{\mathrm{d}}$ & $11.75^{\mathrm{b}}$ & $9.26^{\mathrm{a}}$ \\
\hline & $\mathrm{N} 2$ & $4.76^{\mathrm{ab}}$ & $5.99^{c}$ & $11.15^{\mathrm{b}}$ & $14.21^{\mathrm{c}}$ & $8.98^{c}$ & $9.89^{c}$ & $10.62^{\mathrm{d}}$ & $10.78^{\mathrm{d}}$ & $11.83^{\mathrm{b}}$ & $13.33^{b}$ \\
\hline \multirow[t]{3}{*}{$E\left[\mathrm{mmol}\left(\mathrm{H}_{2} \mathrm{O}\right) \mathrm{m}^{-2} \mathrm{~s}^{-1}\right]$} & No & $2.37^{\mathrm{a}}$ & $2.73^{\mathrm{ab}}$ & $2.27^{\mathrm{a}}$ & $2.85^{\mathrm{ab}}$ & $2.37^{\mathrm{a}}$ & $2.67^{\mathrm{a}}$ & $1.13^{\mathrm{a}}$ & $1.69^{b}$ & $2.45^{\mathrm{a}}$ & $2.14^{\mathrm{a}}$ \\
\hline & N1 & $3.37^{b}$ & $2.51^{\mathrm{a}}$ & $3.03^{\mathrm{b}}$ & $2.34^{\mathrm{a}}$ & $2.78^{\mathrm{a}}$ & $2.59^{\mathrm{a}}$ & $1.56^{\mathrm{b}}$ & $1.76^{\mathrm{b}}$ & $2.50^{\mathrm{a}}$ & $2.30^{\mathrm{a}}$ \\
\hline & $\mathrm{N} 2$ & $2.24^{\mathrm{a}}$ & $2.45^{\mathrm{a}}$ & $2.46^{\mathrm{ab}}$ & $2.30^{\mathrm{a}}$ & $2.41^{\mathrm{a}}$ & $2.30^{\mathrm{a}}$ & $1.51^{\mathrm{b}}$ & $1.60^{\mathrm{b}}$ & $2.11^{\mathrm{a}}$ & $2.46^{\mathrm{a}}$ \\
\hline \multirow[t]{3}{*}{$C_{\mathrm{i}}\left[\mu \mathrm{mol}\left(\mathrm{CO}_{2}\right) \mathrm{mol}^{-1}\right]$} & N0 & $258.3^{\mathrm{a}}$ & $261.3^{\mathrm{a}}$ & $322.0^{\mathrm{a}}$ & $346.7^{\mathrm{a}}$ & $299.0^{\mathrm{a}}$ & $306.0^{\mathrm{a}}$ & $383.0^{\mathrm{c}}$ & $355.0^{\mathrm{b}}$ & $375.0^{\mathrm{a}}$ & $363.0^{\mathrm{a}}$ \\
\hline & N1 & $260.7^{\mathrm{a}}$ & $258,0^{\mathrm{a}}$ & $327.6^{\mathrm{a}}$ & $322.3^{\mathrm{a}}$ & $300.0^{\mathrm{a}}$ & $290.0^{\mathrm{a}}$ & $369.0^{\mathrm{bc}}$ & $339.0^{\mathrm{a}}$ & $373.0^{\mathrm{a}}$ & $357.0^{\mathrm{a}}$ \\
\hline & $\mathrm{N} 2$ & $281.0^{\mathrm{a}}$ & $251.3^{\mathrm{a}}$ & $331.3^{\mathrm{a}}$ & $314.3^{\mathrm{a}}$ & $309.0^{\mathrm{a}}$ & $281.0^{\mathrm{a}}$ & $375.0^{\mathrm{c}}$ & $348.0^{\mathrm{ab}}$ & $361.0^{\mathrm{a}}$ & $353.0^{\mathrm{a}}$ \\
\hline \multirow[t]{3}{*}{$g_{\mathrm{s}}\left[\mathrm{mol}\left(\mathrm{H}_{2} \mathrm{O}\right) \mathrm{m}^{-2} \mathrm{~s}^{-1}\right]$} & No & $0.04^{\mathrm{a}}$ & $0.04^{\mathrm{a}}$ & $0.20^{\mathrm{a}}$ & $0.20^{\mathrm{a}}$ & $0.11^{\mathrm{a}}$ & $0.12^{\mathrm{a}}$ & $0.15^{\mathrm{a}}$ & $0.20^{\mathrm{a}}$ & $0.20^{\mathrm{a}}$ & $0.16^{\mathrm{a}}$ \\
\hline & N1 & $0.04^{\mathrm{a}}$ & $0.05^{\mathrm{a}}$ & $0.21^{\mathrm{a}}$ & $0.17^{\mathrm{a}}$ & $0.12^{\mathrm{a}}$ & $0.11^{\mathrm{a}}$ & $0.25^{\mathrm{a}}$ & $0.23^{\mathrm{a}}$ & $0.33^{\mathrm{b}}$ & $0.14^{\mathrm{a}}$ \\
\hline & $\mathrm{N} 2$ & $0.04^{\mathrm{a}}$ & $0.03^{\mathrm{a}}$ & $0.44^{\mathrm{b}}$ & $0.24^{\mathrm{a}}$ & $0.20^{\mathrm{a}}$ & $0.13^{\mathrm{a}}$ & $0.45^{\mathrm{b}}$ & $0.22^{\mathrm{a}}$ & $0.15^{\mathrm{a}}$ & $0.21^{\mathrm{a}}$ \\
\hline \multirow[t]{3}{*}{ WUE $\left[\mu \mathrm{mol}\left(\mathrm{CO}_{2}\right) \mathrm{mmol}^{-1}\left(\mathrm{H}_{2} \mathrm{O}\right)\right]$} & No & $1.85^{\mathrm{ab}}$ & $1.85^{\mathrm{ab}}$ & $3.62^{\mathrm{ab}}$ & $2.87^{\mathrm{a}}$ & $3.05^{\mathrm{ab}}$ & $2.31^{\mathrm{a}}$ & $3.73^{\mathrm{a}}$ & $3.50^{\mathrm{a}}$ & $3.47^{\mathrm{a}}$ & $3.79^{\mathrm{ab}}$ \\
\hline & $\mathrm{N} 1$ & $1.49^{\mathrm{a}}$ & $2.56^{\mathrm{c}}$ & $3.68^{\mathrm{ab}}$ & $5.47^{\mathrm{cd}}$ & $3.14^{\mathrm{ab}}$ & $3.52^{b c}$ & $5.96^{\mathrm{b}}$ & $5.91^{b}$ & $4.71^{b c}$ & $4.05^{\mathrm{ab}}$ \\
\hline & $\mathrm{N} 2$ & $2.12^{\mathrm{bc}}$ & $2.45^{\mathrm{c}}$ & $4.55^{b c}$ & $6.22^{\mathrm{d}}$ & $3.75^{b c}$ & $4.34^{\mathrm{c}}$ & $7.01^{\mathrm{b}}$ & $6.78^{b}$ & $5.61^{\mathrm{c}}$ & $5.45^{\mathrm{c}}$ \\
\hline
\end{tabular}


Table 3. Two-way ANOVA analysis showing significance of obtained results of morphological plant features ( $\mathrm{SN}$ - shoot number, LN leaf number, RL - total root length, SA - surface area of roots, D - average root diameter, B - biomass of plants, SDM - shoot dry mass, RDM - root dry mass, R/S - root and shoot ratio, SRL - specific root length) relevant to the variety (Felopa, Sulino) and nitrogen dose ( $\mathrm{N} 0$ - no nitrogen, $\mathrm{N} 1$ - single, and $\mathrm{N} 2$ - double dose) in terms of observation (40, 80, and 180 days after sowing, DAS). *, **, *** - significance at the $0.05,0.01$, and 0.001 probability levels, respectively; ns - not significant.

\begin{tabular}{|c|c|c|c|c|c|c|c|c|c|c|c|}
\hline & Term & Pare & neter & & & & & & & & \\
\hline Factors & [DAS] & SN & LN & RL & SA & $\mathrm{D}$ & B & SDM & RDM & $\mathrm{R} / \mathrm{S}$ & SRL \\
\hline Variety & 40 & ns & ns & $* * *$ & $* * *$ & ns & $* *$ & $*$ & $*$ & ns & $* * *$ \\
\hline $\mathrm{N}$ dose & & $* * *$ & ns & $* * *$ & $* * *$ & $*$ & $* * *$ & $*$ & $* * *$ & ns & ns \\
\hline Variety $\times \mathrm{N}$ dose & & $*$ & ns & $* * *$ & $* *$ & ns & $*$ & ns & $*$ & ns & $* * *$ \\
\hline Variety & 80 & ns & ns & * & $* * *$ & ns & ns & ns & ns & ns & ns \\
\hline $\mathrm{N}$ dose & & ns & ns & $* * *$ & $*$ & ns & ns & ns & ns & ns & ns \\
\hline Variety $\times \mathrm{N}$ dose & & ns & ns & $* *$ & * & ns & ns & ns & ns & ns & ns \\
\hline Variety & 180 & $* *$ & $* *$ & $\mathrm{~ns}$ & $\mathrm{~ns}$ & $*$ & ${ }^{*}$ & ${ }^{* *}$ & ns & ns & ns \\
\hline $\mathrm{N}$ dose & & $* * *$ & $* * *$ & $* * *$ & $* * *$ & *** & $* * *$ & $* * *$ & $x_{* * *}$ & ${ }^{*} \mathrm{~s}_{*}$ & $x_{* * *}$ \\
\hline Variety $\times \mathrm{N}$ dose & & $*$ & $* *$ & $* *$ & $* *$ & $* *$ & $* * *$ & $* *$ & $* * *$ & $*$ & $*$ \\
\hline
\end{tabular}

Table 4. Effect of nitrogen dose (N0 - no nitrogen, N1 - single, and N2 - double dose) on morfological plant features (SN - shoot number, LN - leaf number, RL - total root length, SA - surface area of roots, D - average root diameter, B - biomass of plants, SDM - shoot dry mass, RDM - root dry mass, R/S - root and shoot ratio, SRL - specific root length) of festulolium varieties (Felopa, Sulino) in terms of observation (40, 80, and 180 days after sowing, DAS). Mean values in each term of observation (within varieties) followed by the same letter are not significantly different $(p<0.05)$ by the Tukey's HSD test.

\begin{tabular}{|c|c|c|c|c|c|c|c|}
\hline Parameter & $\mathrm{N}$ dose & $\begin{array}{l}40 \text { DAS } \\
\text { Felopa }\end{array}$ & Sulino & $\begin{array}{l}80 \text { DAS } \\
\text { Felopa }\end{array}$ & Sulino & $\begin{array}{l}180 \text { DAS } \\
\text { Felopa }\end{array}$ & Sulino \\
\hline SN [No. per plant] & $\begin{array}{l}\text { N0 } \\
\text { N1 } \\
\text { N2 }\end{array}$ & $\begin{array}{l}1.67^{\mathrm{a}} \\
2.22^{\mathrm{b}} \\
2.00^{\mathrm{ab}}\end{array}$ & $\begin{array}{l}1.67^{\mathrm{a}} \\
2.11^{\mathrm{ab}} \\
2.28^{\mathrm{b}}\end{array}$ & $\begin{array}{l}4.00^{\mathrm{a}} \\
4.22^{\mathrm{a}} \\
3.67^{\mathrm{a}}\end{array}$ & $\begin{array}{l}4.94^{\mathrm{a}} \\
4.67^{\mathrm{a}} \\
4.25^{\mathrm{a}}\end{array}$ & $\begin{array}{l}9.89^{\mathrm{a}} \\
13.08^{\mathrm{ab}} \\
16.58^{\mathrm{bc}}\end{array}$ & $\begin{array}{l}11.17^{\mathrm{a}} \\
16.17^{\mathrm{bc}} \\
20.42^{\mathrm{c}}\end{array}$ \\
\hline LN [No. per plant] & $\begin{array}{l}\text { N0 } \\
\text { N1 } \\
\text { N2 }\end{array}$ & $\begin{array}{l}5.89^{\mathrm{a}} \\
5.94^{\mathrm{a}} \\
6.33^{\mathrm{a}}\end{array}$ & $\begin{array}{l}5.33^{\mathrm{a}} \\
7.11^{\mathrm{a}} \\
6.31^{\mathrm{a}}\end{array}$ & $\begin{array}{l}13.89^{\mathrm{a}} \\
15.83^{\mathrm{a}} \\
12.78^{\mathrm{a}}\end{array}$ & $\begin{array}{l}15.33^{\mathrm{a}} \\
15.89^{\mathrm{a}} \\
13.42^{\mathrm{a}}\end{array}$ & $\begin{array}{l}15.42^{\mathrm{a}} \\
31.92^{\mathrm{b}} \\
44.75^{\mathrm{cd}}\end{array}$ & $\begin{array}{l}16.00^{\mathrm{a}} \\
40.25^{\mathrm{bc}} \\
52.67^{\mathrm{d}}\end{array}$ \\
\hline $\mathrm{RL}$ [cm per plant] & $\begin{array}{l}\text { N0 } \\
\text { N1 } \\
\text { N2 }\end{array}$ & $\begin{array}{l}110.7^{\mathrm{a}} \\
146.0^{\mathrm{b}} \\
109.3^{\mathrm{a}}\end{array}$ & $\begin{array}{l}127.8^{\mathrm{ab}} \\
257.4^{\mathrm{d}} \\
195.4^{\mathrm{c}}\end{array}$ & $\begin{array}{l}436.9^{\mathrm{b}} \\
518.6^{\mathrm{cd}} \\
357.2^{\mathrm{a}}\end{array}$ & $\begin{array}{l}466.9^{\mathrm{bc}} \\
490.9^{\mathrm{bc}} \\
446.9^{\mathrm{bc}}\end{array}$ & $\begin{array}{l}1,454.0^{\mathrm{cd}} \\
900.5^{\mathrm{ab}} \\
829.7^{\mathrm{a}}\end{array}$ & $\begin{array}{l}1,375.4^{\mathrm{c}} \\
954.6^{\mathrm{b}} \\
855.1^{\mathrm{ab}}\end{array}$ \\
\hline $\mathrm{SA}\left[\mathrm{cm}^{2}\right]$ & $\begin{array}{l}\text { N0 } \\
\text { N1 } \\
\text { N2 }\end{array}$ & $\begin{array}{l}15.24^{\mathrm{a}} \\
21.41^{\mathrm{b}} \\
13.23^{\mathrm{a}}\end{array}$ & $\begin{array}{l}13.57^{\mathrm{a}} \\
26.99^{\mathrm{c}} \\
21.32^{\mathrm{b}}\end{array}$ & $\begin{array}{l}47.18^{\mathrm{ab}} \\
42.54^{\mathrm{a}} \\
40.34^{\mathrm{a}}\end{array}$ & $\begin{array}{l}65.40^{\mathrm{b}} \\
59.47^{\mathrm{b}} \\
52.38^{\mathrm{ab}}\end{array}$ & $\begin{array}{l}413.89^{\mathrm{d}} \\
245.72^{\mathrm{ab}} \\
199.35^{\mathrm{a}}\end{array}$ & $\begin{array}{l}351.59^{\mathrm{cd}} \\
314.78^{\mathrm{bc}} \\
189.60^{\mathrm{a}}\end{array}$ \\
\hline $\mathrm{D}[\mathrm{mm}]$ & $\begin{array}{l}\text { N0 } \\
\text { N1 } \\
\text { N2 }\end{array}$ & $\begin{array}{l}0.39^{\mathrm{a}} \\
0.43^{\mathrm{a}} \\
0.35^{\mathrm{a}}\end{array}$ & $\begin{array}{l}0.36^{\mathrm{a}} \\
0.41^{\mathrm{a}} \\
0.38^{\mathrm{a}}\end{array}$ & $\begin{array}{l}0.44^{\mathrm{a}} \\
0.40^{\mathrm{a}} \\
0.48^{\mathrm{a}}\end{array}$ & $\begin{array}{l}0.45^{\mathrm{a}} \\
0.44^{\mathrm{a}} \\
0.45^{\mathrm{a}}\end{array}$ & $\begin{array}{l}0.94^{\mathrm{a}} \\
0.89^{\mathrm{a}} \\
0.82^{\mathrm{a}}\end{array}$ & $\begin{array}{l}0.96^{\mathrm{a}} \\
1.19^{\mathrm{b}} \\
0.76^{\mathrm{a}}\end{array}$ \\
\hline B [g(DM) per plant] & $\begin{array}{l}\text { N0 } \\
\text { N1 } \\
\text { N2 }\end{array}$ & $\begin{array}{l}0.082^{\mathrm{a}} \\
0.112^{\mathrm{ab}} \\
0.085^{\mathrm{ab}}\end{array}$ & $\begin{array}{l}0.092^{\mathrm{a}} \\
0.121^{\mathrm{b}} \\
0.111^{\mathrm{a}}\end{array}$ & $\begin{array}{l}0.386^{\mathrm{a}} \\
0.428^{\mathrm{a}} \\
0.346^{\mathrm{a}}\end{array}$ & $\begin{array}{l}0.417^{\mathrm{a}} \\
0.439^{\mathrm{a}} \\
0.415^{\mathrm{a}}\end{array}$ & $\begin{array}{l}1.732^{\mathrm{b}} \\
2.220^{\mathrm{c}} \\
2.306^{\mathrm{c}}\end{array}$ & $\begin{array}{l}1.517^{\mathrm{a}} \\
2.474^{\mathrm{c}} \\
2.553^{\mathrm{c}}\end{array}$ \\
\hline SDM [g(DM) per plant] & $\begin{array}{l}\text { N0 } \\
\text { N1 } \\
\text { N2 }\end{array}$ & $\begin{array}{l}0.055^{\mathrm{a}} \\
0.071^{\mathrm{a}} \\
0.053^{\mathrm{a}}\end{array}$ & $\begin{array}{l}0.059^{\mathrm{a}} \\
0.075^{\mathrm{a}} \\
0.075^{\mathrm{a}}\end{array}$ & $\begin{array}{l}0.275^{\mathrm{a}} \\
0.293^{\mathrm{a}} \\
0.251^{\mathrm{a}}\end{array}$ & $\begin{array}{l}0.296^{\mathrm{a}} \\
0.311^{\mathrm{a}} \\
0.296^{\mathrm{a}}\end{array}$ & $\begin{array}{l}0.636^{\mathrm{a}} \\
1.360^{\mathrm{b}} \\
1.451^{\mathrm{b}}\end{array}$ & $\begin{array}{l}0.557^{\mathrm{a}} \\
1.470^{\mathrm{b}} \\
1.717^{\mathrm{c}}\end{array}$ \\
\hline RDM [g(DM) per plant] & $\begin{array}{l}\text { N0 } \\
\text { N1 } \\
\text { N2 }\end{array}$ & $\begin{array}{l}0.027^{\mathrm{a}} \\
0.041^{\mathrm{bc}} \\
0.032^{\mathrm{ab}}\end{array}$ & $\begin{array}{l}0.033^{\mathrm{ab}} \\
0.046^{\mathrm{c}} \\
0.037^{\mathrm{abc}}\end{array}$ & $\begin{array}{l}0.111^{\mathrm{a}} \\
0.135^{\mathrm{a}} \\
0.095^{\mathrm{a}}\end{array}$ & $\begin{array}{l}0.121^{\mathrm{a}} \\
0.128^{\mathrm{a}} \\
0.119^{\mathrm{a}}\end{array}$ & $\begin{array}{l}1.096^{\mathrm{b}} \\
0.860^{\mathrm{a}} \\
0.855^{\mathrm{a}}\end{array}$ & $\begin{array}{l}1.013^{\mathrm{b}} \\
1.004^{\mathrm{b}} \\
0.836^{\mathrm{a}}\end{array}$ \\
\hline $\mathrm{R} / \mathrm{S}\left[\mathrm{g} \mathrm{g}^{-1}\right]$ & $\begin{array}{l}\text { N0 } \\
\text { N1 } \\
\text { N2 }\end{array}$ & $\begin{array}{l}0.50^{\mathrm{a}} \\
0.60^{\mathrm{a}} \\
0.61^{\mathrm{a}}\end{array}$ & $\begin{array}{l}0.56^{\mathrm{a}} \\
0.61^{\mathrm{a}} \\
0.49^{\mathrm{a}}\end{array}$ & $\begin{array}{l}0.40^{\mathrm{a}} \\
0.47^{\mathrm{a}} \\
0.38^{\mathrm{a}}\end{array}$ & $\begin{array}{l}0.41^{\mathrm{a}} \\
0.41^{\mathrm{a}} \\
0.40^{\mathrm{a}}\end{array}$ & $\begin{array}{l}1.72^{\mathrm{c}} \\
0.64^{\mathrm{b}} \\
0.59^{\mathrm{ab}}\end{array}$ & $\begin{array}{l}1.82^{\mathrm{c}} \\
0.69^{\mathrm{b}} \\
0.49^{\mathrm{a}}\end{array}$ \\
\hline $\mathrm{SRL}\left[\mathrm{m} \mathrm{g}^{-1}\right]$ & $\begin{array}{l}\text { N0 } \\
\text { N1 } \\
\text { N2 }\end{array}$ & $\begin{array}{l}41.29^{\mathrm{a}} \\
35.56^{\mathrm{a}} \\
34.10^{\mathrm{a}}\end{array}$ & $\begin{array}{l}39.46^{\mathrm{a}} \\
56.94^{\mathrm{b}} \\
53.61^{\mathrm{b}}\end{array}$ & $\begin{array}{l}39.70^{\mathrm{a}} \\
38.76^{\mathrm{a}} \\
38.65^{\mathrm{a}}\end{array}$ & $\begin{array}{l}39.01^{\mathrm{a}} \\
38.45^{\mathrm{a}} \\
38.53^{\mathrm{a}}\end{array}$ & $\begin{array}{l}13.27^{\mathrm{b}} \\
10.49^{\mathrm{a}} \\
9.71^{\mathrm{a}}\end{array}$ & $\begin{array}{l}13.58^{\mathrm{b}} \\
9.58^{\mathrm{a}} \\
10.27^{\mathrm{a}}\end{array}$ \\
\hline
\end{tabular}




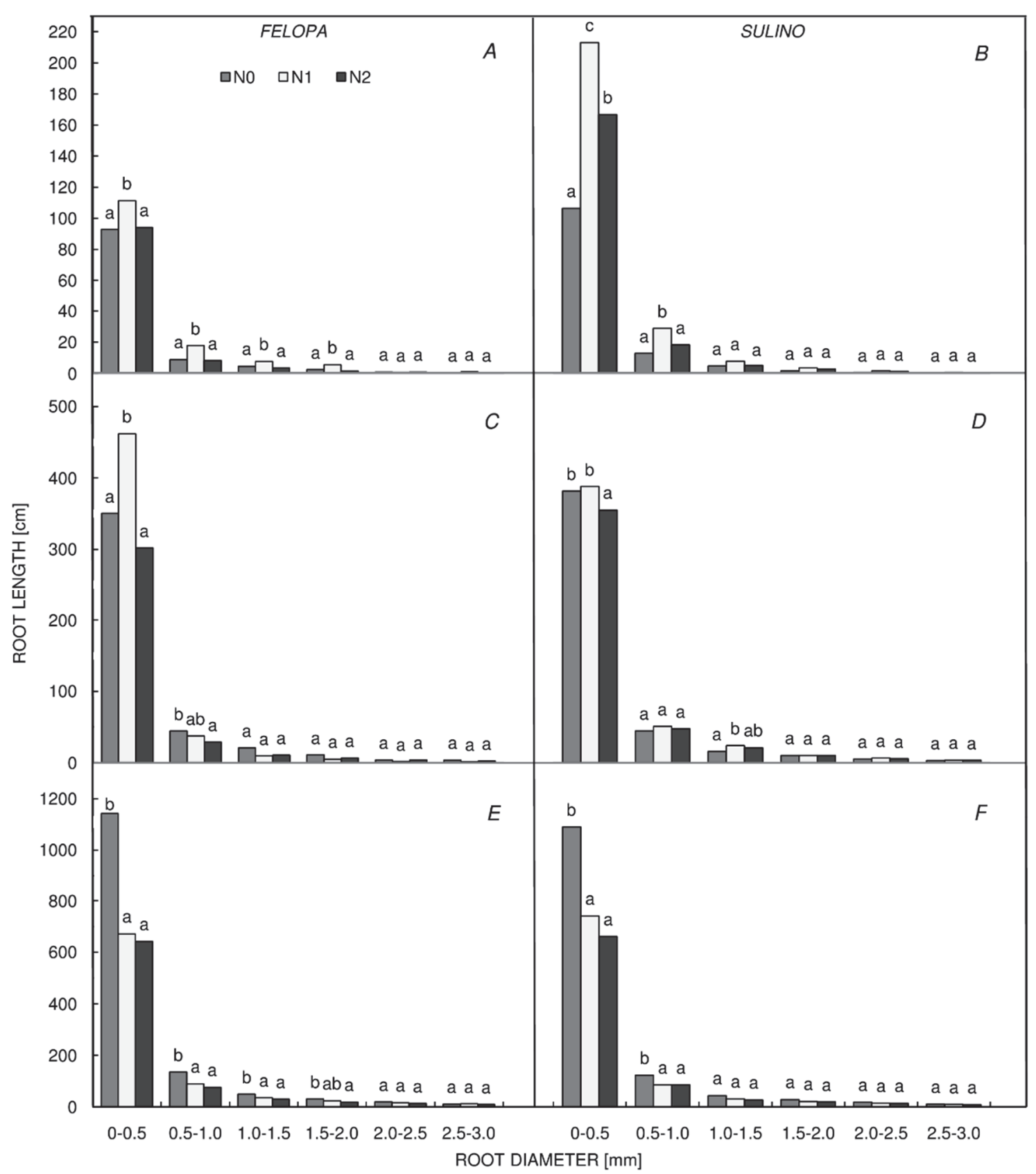

Fig. 1. Diameter distribution of festulolium varieties (Felopa and Sulino) under the influence of different nitrogen application: no nitrogen (N0), single (N1), and double (N2) dose in terms of observation $(A, B-40 ; C, D-80$, and $E, F-180$ days after sowing). Columns followed by the same letter are not significantly different $(p<0.05)$ by the Tukey's HSD test.

affected by the variety and the dosage of nitrogen (Table 3). The effect of $\mathrm{N}$ fertilization on shoot (SN) and leaf (LN) number was visible only at the end of the investigation (180 DAS), especially at the DD of $\mathrm{N}$ (Tables 3, 4). It was found, that the Sulino variety formed more shoots than that of the Felopa variety, regardless of fertilization, at the $80 \mathrm{DAS}$, from sowing to the end of the study period. The effect of $\mathrm{N}$ fertilization on the total root length (RL) of festulolium varieties depended on the tillering intensity of plants. At the first (40 DAS) and second (80 DAS) terms of observation, the SD $\mathrm{N}$ dose positively affected RL, while at the end of the study, plants were characterized by the longest roots under conditions without $\mathrm{N}$ fertilization (from $1,373.3$ to $1,454.0 \mathrm{~cm}$ per plant). Regardless of the term of observation, the RL of plant varieties was the lowest at the DD N dose (Tables 3,4). The effect of $\mathrm{N}$ fertilization on the surface area (SA) of plant roots was noticed at 40 DAS. SD $\mathrm{N}$ dose positively affected SA in this term, whereas, in subsequent terms, the greatest SA was reported in the absence of $\mathrm{N}$ fertilization. A greater SA, especially at the beginning of investigation, was characteristic for the tested Sulino variety. 


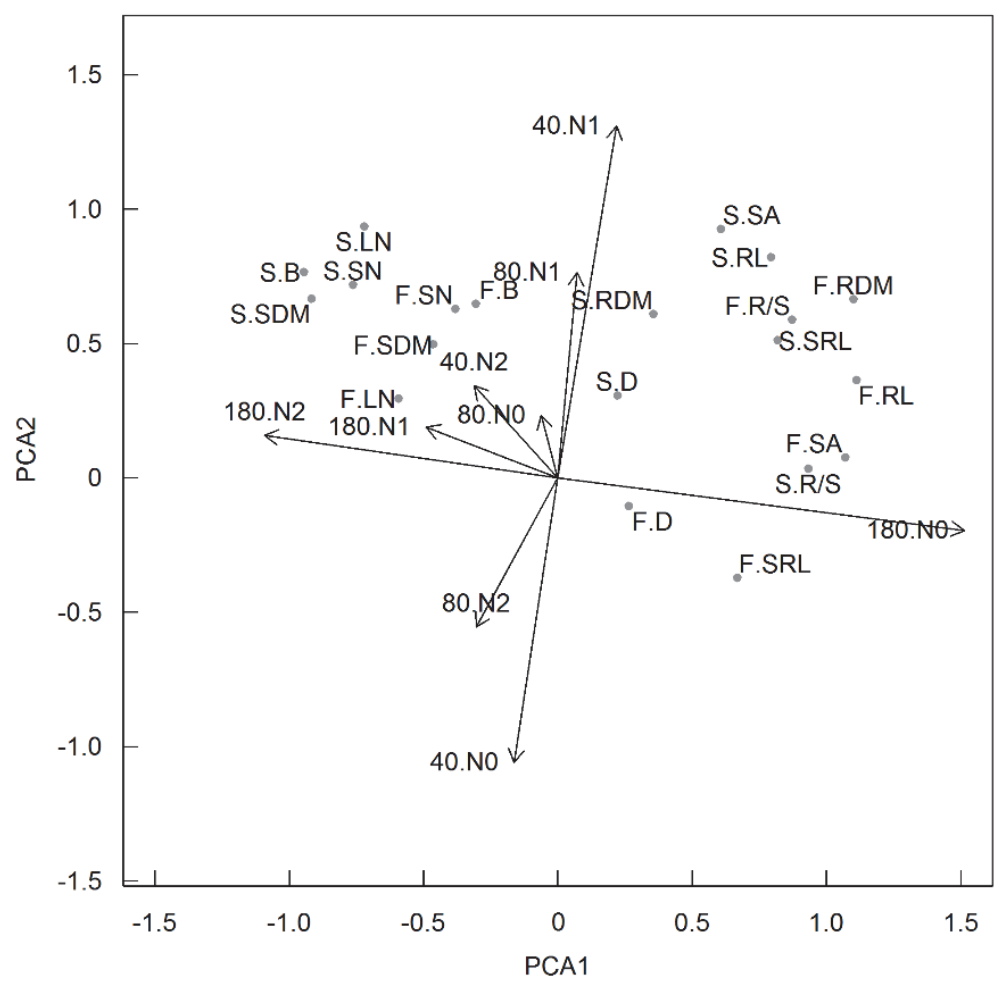

Fig. 2. Principle component analysis (PCA) bi-plot for two-way classification of variety-by-features combination (first factor) and termby-nitrogen dose combination (second factor); varieties: Felopa (F), Sulino (S); plant features: SN - shoot number, LN - leaf number, $\mathrm{RL}$ - total root length, SA - surface area of roots, D - average root diameter, B - biomass of plants, SDM - shoot dry mass, $\mathrm{RDM}$ - root dry mass, R/S - root and shoot ratio, SRL - specific root length; terms of observation: after 40, 80, 180 days from sowing; nitrogen application: $\mathrm{N} 0$ - no nitrogen, $\mathrm{N} 1$ - single, and N2 - double dose.

Table 5. Correlation coefficients $(r)$ between physiological parameters (SPAD - leaf greenness index, $P_{\mathrm{N}}-$ net photosynthetic rate, $E$ - transpiration rate, $C_{\mathrm{i}}$ - intercellular $\mathrm{CO}_{2}$ concentration, $g_{\mathrm{s}}$ - stomatal conductance, WUE - water-use efficiency) and morphological traits (SN - shoot number, LN - leaf number, RL - total root length, SA - surface area of roots, D - average root diameter, SDM - shoot dry mass, RDM - root dry mass, R/S - root and shoot ratio, SRL - specific root length) of festulolium plants. The coefficient values marked by $* * *$ are significant at $p<0.05$ and $p<0.01$, respectively. Values are means for both tested varieties.

\begin{tabular}{lllllllllllllll}
\hline Variable & $P_{\mathrm{N}}$ & $E$ & $C_{\mathrm{i}}$ & $g_{\mathrm{s}}$ & WUE & $\mathrm{SN}$ & $\mathrm{LN}$ & $\mathrm{RL}$ & $\mathrm{SA}$ & $\mathrm{D}$ & $\mathrm{SDM}$ & $\mathrm{RDM}$ & $\mathrm{R} / \mathrm{S}$ & $\mathrm{SRL}$ \\
\hline SPAD & $0.84^{* *}$ & 0.07 & -0.31 & 0.19 & $0.81^{* *}$ & $0.69^{* *}$ & $0.85^{* *}$ & $-0.95^{* *}$ & $-0.81^{* *}$ & -0.22 & $0.93^{* *}$ & $-0.67^{* *}$ & $-0.96^{* *}$ & $-0.87^{* *}$ \\
$P_{\mathrm{N}}$ & 1 & 0.27 & -0.26 & 0.33 & $0.85^{* *}$ & $0.66^{* *}$ & $0.78^{* *}$ & $-0.81^{* *}$ & $-0.83^{* *}$ & $-0.52^{*}$ & $0.80^{* *}$ & $-0.82^{* *}$ & $-0.82^{* *}$ & $-0.61^{* *}$ \\
$E$ & - & 1 & 0.44 & $0.64^{* *}$ & -0.28 & -0.07 & 0.02 & -0.08 & 0.10 & 0.01 & 0.12 & 0.09 & -0.09 & -0.18 \\
$C_{\mathrm{i}}$ & - & - & 1 & $0.61^{* *}$ & -0.46 & $-0.63^{* *}$ & $-0.55^{*}$ & 0.35 & 0.42 & 0.06 & -0.46 & 0.39 & 0.40 & 0.21 \\
$g_{\mathrm{S}}$ & - & - & - & 1 & -0.01 & -0.12 & -0.04 & -0.20 & -0.16 & -0.18 & 0.10 & -0.21 & -0.17 & -0.15 \\
WUE & - & - & - & - & 1 & $0.70^{* *}$ & $0.77^{* *}$ & $-0.77^{* *}$ & $-0.89^{* *}$ & $-0.54^{*}$ & $0.73^{* *}$ & $-0.85^{* *}$ & $-0.77^{* *}$ & $-0.53^{*}$ \\
SN & - & - & - & - & - & 1 & $0.96^{* *}$ & $-0.71^{* *}$ & $-0.76^{* *}$ & -0.35 & $0.85^{* *}$ & $-0.61^{* *}$ & $-0.78^{* *}$ & $-0.58^{*}$ \\
LN & - & - & - & - & - & - & 1 & $-0.86^{* *}$ & $-0.82^{* *}$ & -0.29 & $0.94^{* *}$ & $-0.66^{* *}$ & $-0.91^{* *}$ & $-0.75^{* *}$ \\
RL & - & - & - & - & - & - & - & 1 & $0.86^{* *}$ & 0.15 & $-0.93^{* *}$ & $0.70^{* *}$ & $0.97^{* *}$ & $0.90^{* *}$ \\
SA & - & - & - & - & - & - & - & - & 1 & $0.55^{*}$ & $-0.80^{* *}$ & $0.82^{* *}$ & $0.83^{* *}$ & $0.66^{* *}$ \\
D & - & - & - & - & - & - & - & - & - & 1 & -0.21 & $0.55^{*}$ & 0.20 & -0.08 \\
SDM & - & - & - & - & - & - & - & - & - & - & 1 & $-0.63^{* *}$ & $-0.98^{* *}$ & $-0.86^{* *}$ \\
RDM & - & - & - & - & - & - & - & - & - & - & - & 1 & $0.70^{* *}$ & 0.34 \\
R/S & - & - & - & - & - & - & - & - & - & - & - & - & 1 & $0.86^{* *}$ \\
\hline
\end{tabular}

The influence of the $\mathrm{N}$ application on the average diameter of roots (D) was found only in the final period of the study (180 DAS) in the Sulino variety. A SD N dose significantly increased the root diameter $(1.19 \mathrm{~mm})$ in comparison to DD $(0.76 \mathrm{~mm})$. Distribution of root length according to classes defined by their diameter varied in all terms of observation depending on the nitrogen applied (Fig. 1A-F). The finest roots were in the range of $0-0.5 \mathrm{~mm}$ in diameter, irrespective of the factors investigated. It was noted that at the beginning of tillering (40 DAS) a SD N 
dose, regardless of the plant variety, increased root length significantly in classes separated according to a diameter, while a DD reduced their length (Fig. $1 A, B)$. In the last period of study (180 DAS), festulolium plants with no $\mathrm{N}$ application had significantly longer roots in separated classes compared to those of fertilized plants. At the same time, increasing the $\mathrm{N}$ dose caused the formation of shorter roots in all classes defined by a diameter (Fig. 1E,F).

The total plant biomass (B) was the smallest with no $\mathrm{N}$ application, which was determined by the small SN and SDM formed by varieties tested in relation to the objects fertilized (Table 4). A DD of $\mathrm{N}$ adversely affected the RDM compared to a SD of $\mathrm{N}$ (especially at 40 and 80 DAS) and the absence of fertilization (180 DAS). A significant influence of the $\mathrm{N}$ application on the $\mathrm{R} / \mathrm{S}$ ratio was found only at the end of the study (180 DAS). The highest values of the R/S ratio were obtained with no $\mathrm{N}$ fertilization (Table 4). It was caused by the mass of roots increasing during this period. The results also showed a gradual reduction in the $\mathrm{R} / \mathrm{S}$ ratio under increasing $\mathrm{N}$ fertilization due to a large SN and SDM of both plant varieties. Values of the specific root length (SRL) of plants tested varied in terms of observation, depending on the $\mathrm{N}$ application. In the initial period of the study the Sulino variety obtained a higher value of SRL when compared to the Felopa variety, for both $\mathrm{N}$ doses (SD and DD). Whereas, in the final period (180 DAS) SRL, similarly as the $\mathrm{R} / \mathrm{S}$ ratio of varieties, decreased significantly with $\mathrm{N}$ fertilization.

Principle component analysis (PCA) showed that the first component (PC1) retained $42.0 \%$ of the sum of squares for standardized data set and the second (PC2) $25.7 \%$, respectively (Fig. 2). The features grouped in the

\section{Discussion}

Chl concentration is a trait indicating the nitrogen status of plants. There is a close relationship between the $\mathrm{N}$ content in plant leaves and SPAD readings, as well (Gáborčík 2003). Chl can also be considered an indicator of the annual grass yield, since a positive correlation exists between its content in the leaf blade of grasses and its dry matter yield (Olszewska 2008b). In our study the values of the SPAD index increased under a higher $\mathrm{N}$ dose at all terms of observation. Lower values in the leaf greenness index were found in the Sulino variety (by about 6.5\%). These results are consistent with those of Olszewska (2008b), who showed that Sulino grown as a pure strain was characterized by lower values of SPAD than the Felopa variety (by 6.1\%). She reported that nitrogen fertilization affected the leaf greenness index and the highest SPAD values were found in plants fertilized with the highest dose of nitrogen (120 kg ha- per year). According to Shangguan et al. (2000), a nitrogen content in leaves is in close correlation with the content of Rubisco, which is the most common enzyme in the leaves of $\mathrm{C}_{3}$ plants, and plays an important role in $\mathrm{CO}_{2}$ plot in two clusters; those connected with the aboveground part of plants were placed in the upper-left side of the plot, while those being connected with the roots were on the upper-right side. Thus, the response of aboveground part of plants and roots to the environmental conditions was different. The division of plants into above- and belowground parts gave us the information about the impact of nitrogen dose and the term of its application on the mass allocation in plants. It was found, that under conditions without the $\mathrm{N}$ application the features examined were lower in comparison to SD and DD N dose in the first term, 40 DAS (except D and SRL values for Felopa variety). The tested varieties, especially the Sulino, invested in their aboveground part and roots till 80 DAS. In the next period, the plants invested strictly into their roots, and the Felopa variety showed greater growth particularly for SA, RL, and RDM features, than the Sulino variety.

The values of root features were higher for SD N dose at the beginning of the tillering phase in comparison with the DD. All plant features, except D and SRL, for both varieties were higher than mean values for respective feature-by-term combinations. SD $\mathrm{N}$ dose in the first (40 DAS) and second (80 DAS) terms of observation affected each in a similar way, so plants invested in the same root features. Whereas, at the end of study, the plants strictly invested into the aboveground part (Sulino more than Felopa). The results showed that under DD N dose, in the first term of observation, the higher values were noted for the aboveground features as well. In the next term, the values of above- and belowground features were lower, while in the third term, the response of varieties were the same as in the first term.

assimilation. Research conducted by Olszewska (2008a) on festulolium (Festulolium braunii) and Grygierzec (2012) on perennial rye grass (Lolium perenne) showed that the highest rate of plant photosynthesis was recorded at a medium dose of nitrogen (50-60 kg ha ${ }^{-1}$ per year) and the intensity in this process was reduced in plants fertilized with a double $\mathrm{N}$ dose (100-120 kg ha-1 per year). According to Chen et al. (2005), varying $\mathrm{N}$ addition rates do not alter the gas-exchange characteristics of grasses (Leymus chinensis and Stipa grandis) including the $P_{\mathrm{N}}, E$, $g_{\mathrm{s}}$, and $C_{\mathrm{i}}$. The results obtained confirmed that $P_{\mathrm{N}}$ depended on the $\mathrm{N}$ availability. Lack of nitrogen in growth medium affected negatively this process. No difference were found in $P_{\mathrm{N}}$ between plants treated with a single or a double dose of nitrogen. Nitrogen application increased the values of $g_{\mathrm{s}}$ in Felopa variety plants, especially at 80 and 135 DAS. Other studies report contradictory conclusions about the effect of $\mathrm{N}$ deficiency on $g_{\mathrm{s}}$. Lower values of $g_{\mathrm{s}}$ have been reported by Broadley et al. (2000), Shangguan et al. (2000), and Zhu et al. (2014), but the opposite or no influence of the $\mathrm{N}$ application is also 
registered (Freedeen et al. 1991, Ciompi et al. 1996). WUE determines the water relations of plants. In our study, nitrogen fertilization influenced the better use of water by plants. An increase in WUE in response to nitrogen fertilization of festulolium variety plants has been observed previously by Olszewska (2008a).

Increased root length (RL) is an important feature for the acquisition of nutrients at their low availability. Data presented were in accordance with the results of studies carried out by Ryser and Lampers (1995), who found that orchard grass (Dactylis glomerata) responded to an increasing $\mathrm{N}$ supply by decreasing root length. A similar trend in the reduction of RL at a double $\mathrm{N}$ dose $[200 \mathrm{~kg}(\mathrm{~N})$ $\mathrm{ha}^{-1}$ per year] was observed on winter wheat by Svoboda and Haberle (2006).

In our study, the $\mathrm{N}$ application affected the average diameter of roots (D) in the variety Sulino only in the final period of the experiment. Simultaneously, plants with no nitrogen had significantly longer roots in defined classes when compared to the fertilized plants. At the same time, increasing the $\mathrm{N}$ dose caused the formation of shorter roots in all separated diameter classes. Ryser and Lamber (1995) investigated fast- and slow-growing grasses and revealed that their roots became finer with a decreasing nutrient supply. The frequency in distribution of the root diameter showed a peak of fine roots below $0.2-0.3 \mathrm{~mm}$, and with an increasing nutrient supply this peak shifted towards larger diameters (about $1 \mathrm{~mm}$ ).

In this experiment, the influence of the $\mathrm{N}$ dose on plant biomass was visible in the last period of study. The total plant biomass (B) and SDM were the smallest with no $\mathrm{N}$ application in relation to fertilized plants. At this time a double $\mathrm{N}$ dose adversely affected the RDM when compared to the lack of fertilization. A large share of RDM in $\mathrm{B}$ in the absence of nitrogen fertilization increased the $\mathrm{R} / \mathrm{S}$ ratio as well. This data is supported by Ryser and Lampers (1995) and Fiala (2010), who found that with a decreasing supply of nutrients, especially nitrogen, plants allocate proportionally more of their biomass into the roots, which become finer. The results obtained here also corresponded with the findings of Holub et al. (2013). The authors showed that an $\mathrm{N}$ application of $90 \mathrm{~kg} \mathrm{ha}^{-1}$ per year resulted in greater root productivity in comparison with objects unfertilized and fertilized with a double $\mathrm{N}$ dose (180 $\mathrm{kg} \mathrm{ha}^{-1}$ per year). Boot and Mensink (1990) reported that plants growing at low nitrogen supply conditions were characterized by a significant increase in their $\mathrm{R} / \mathrm{S}$ ratio.

Values of the specific root length (SRL) of tested plants varied during the study period and decreased with $\mathrm{N}$ fertilization, especially at the end of the study. A decrease in SRL values with fertilization and, particularly, high SRL in plants under nutrient shortage have also been observed by Ostonen et al. (2007), who argued that it is a good indicator of environmental change for tree species. Leuschner et al. (2013) detected a positive SRL response to fertilization (NPK) only in the dicot species, while grasses showed neither a significant response nor a trend towards a higher value in fertilized compared to unfertilized plants. In the study of Gastal et al. (2010), the species of grass exhibited a contrasting and differing response SRL to a high and low N supply. Red fescue (Festuca rubra), for example, was characterized by the greatest SRL values, while tall fescue (Festuca arundinacea) showed the smallest ones. This was in agreement with earlier studies showing that species from a nutrientpoor environment generally have a higher SRL and finer roots (Boot and Mensink, 1990).

The response of morphological parameters in festulolium to the nitrogen applications varied during the study period. For a better understanding of the $\mathrm{N}$ dose effect on these features, principal component analysis was applied. Application of PCA enables separate parameters according to changes in plant physiological state depending on nutrient deficiency (Kalaji et al. 2014). This approach in our study allowed transforming the set of measured parameters and grouped them in two subclasses: connected with the aboveground part of plants and connected with the roots. The aboveground features of festulolium plants during the tillering phase positively responded to $\mathrm{N}$ doses, whereas without $\mathrm{N}$ fertilization, the plants invested strictly into their roots. Our results were in agreement with Gastal et al. (2010) who stated that relative biomass allocations to roots decreased with increasing $\mathrm{N}$ supply for forage grass species.

A comparison of the correlation coefficients related to studied physiological parameters $\left(P_{\mathrm{N}}, \mathrm{SPAD}\right.$, and WUE) showed a significant relationship between them (Table 5). Higher values of $P_{\mathrm{N}}$ were associated with a higher Chl content (SPAD index) and better use of water by plants. The studied morphological features (LN, SN, SDM, RDM, and RL) of the plants tested were positively correlated with each other as well, especially in the Felopa variety. Photosynthesis is a process that affects the production of plant biomass (Kalaji and Nalborczyk 1993), however, its high intensity expressed per unit of area does not always determine an equal growth of all plant parts. Our results showed that, in both varieties, there was a positive dependence between $\mathrm{CO}_{2}$ assimilation and morphological traits with regards to the aboveground parts of plants (LN, $\mathrm{SN}$, and SDM) and a negative dependence to the belowground parts (RDM and RL). An increased allocation of dry matter to the roots in response to nitrogen deficiency/ low amount in growth medium was associated with a reduction in $\mathrm{CO}_{2}$ assimilation, WUE, the leaf greenness index, and leaf development. Simultaneously, there was no clear indication showing that $E, g_{\mathrm{s}}$, and $C_{\mathrm{i}}$ values of both festulolium varieties were directly related to changes in the morphological features of above- and belowground parts of plants. Mineral deficiency leads to a decrease in growth and accumulation of biomass, which is associated with downregulation due to lower demand for assimilates. Supply of nutrients to acceptors (roots and leaves) performing different physiological functions is tightly regulated and dependent not only on the stage of ontogeny 
of the plants, but also on environmental conditions. Under deficiency of nutrients, priority in the supply have organs protecting plant in the greatest extent from disorders of homeostasis of the whole plant (Starck 2010). In the case of forage grasses, this role is played by the roots. Allocation between root and shoots is an adaptative trait in addition to other shoot and root features (Gastal et al. 2010).

Conclusions: The lack of a nitrogen in growth medium lowered the leaf greenness index (SPAD), $\mathrm{CO}_{2}$ assimilation, and the photosynthetic water-use efficiency of festulolium plants, resulting in a lower dry mass of aboveground parts. Nitrogen fertilization had a strong influence on the growth and biomass of both varieties investigated. The aboveground features of festulolium plants during the tillering phase positively responded to $\mathrm{N}$ doses, while the root features were higher without $\mathrm{N}$

\section{References}

Boller B., Posselt U.K., Veronesi F. (ed.): Fodder Crops and Amenity Grasses. Pp. 523. Springer, New York 2010.

Boot R.G.A., Mensink M.: Size and morphology of root systems of perennial grasses from contrasting habits as affected by nitrogen supply. - Plant Soil 129: 291-299, 1990.

Broadley M.R., Escobar-Gutiérrez A.J., Burns A., Burns I.G.: What are the effects of nitrogen deficiency on growth components of lettuce? - New Phytol. 147: 519-526, 2000.

Chen S., Bai Y., Zhang L. et al.: Comparing physiological responses of two dominant grass species to nitrogen addition in Xilin River Basin of China. - Environ. Exp. Bot. 53: 65-75, 2005.

Ciompi S., Gentili E., Guidi L. et al.: The effect of nitrogen deficiency on leaf gas exchange and chlorophyll fluorescence parameters in sunflower. - Plant Sci. 118: 177-184, 1996.

Fiala K.: Below ground plant biomass of grassland ecosystems and its variation according to ecological factors. - Ekol.Bratislava 29: 182-206, 2010.

Fox J., Weisberg S.: An $R$ Companion to Applied Regression. $2^{\text {nd }}$ ed. Pp. 472. Sage Publ. Inc., Thousand Oaks, 2011

Fredeen A.L., Gamon J.A., Field C.B.: Responses of photosynthesis and carbohydrate-partitioning to limitations in nitrogen and water availability in field-grown sunflower. Plant Cell Environ. 14: 963-970, 1991.

Gáborčík N.: Relationship between contents of chlorophyll $(a+b)$ (SPAD values) and nitrogen of some temperate grasses. Photosynthetica 41: 285-287, 2003.

Gastal F., Dawson L.A., Thornton B.: Responses of plant traits of four grasses from contrasting habitats to defoliation and $\mathrm{N}$ supply. - Nutr. Cycl. Agroecosys. 88: 245-258, 2010.

Grygierzec B.: Productivity of selected grasses in mixtures with Trifolium repens L. at two levels of nitrogen fertilization. Fragm. Agron. 29: 31-36, 2012.

Gutmane I., Adamovics A..: Influence of nitrogen fertilization rates on Festulolium and Lolium $\times$ boucheanum forage yield and persistency. - Grassland Sci. Eur. 14: 336-338, 2009.

Hill J.O., Simpson R.J., Moore A.D. et al.: Morphology and response of roots of pasture species to phosphorus and nitrogen nutrition. - Plant Soil 286: 7-19, 2006.

Hodge A., Berta G., Doussan C. et al.: Plant root growth, fertilization. Nitrogen application caused the formation of shorter roots in all classes defined by diameter and lowered values of the $\mathrm{SRL}$ and $\mathrm{R} / \mathrm{S}$ ratios. Under conditions without nitrogen, festulolium plants formed longer and finer roots.

In this study, for the first time, we revealed the dependence between different morphological and physiological features at both shoot and root levels in grass plants growing under nitrogen deficiency. A reliance between physiological parameters related to photosynthetic activity of plants $\left(P_{\mathrm{N}}, \mathrm{SPAD}\right.$, and WUE) and morphological traits was positive regarding the aboveground parts of plants (LN, SN, and SDM) and negative regarding the belowground parts (RDM, SA, D, and RL). This could be explained as a survival mechanism for plants under nitrogen deficiency, where the investment of new produced biomass is directed to build up the roots which can improve further access to nitrogen in soil.

architecture and function. - Plant Soil 321: 153-187, 2009.

Holub B., Tůma K., Fiala K.: Effect of fertilization on root growth in the wet submontane meadow. - Plant Soil Environ. 8: 342-347, 2013.

James J.J.: Leaf nitrogen productivity as a mechanism driving the success of invasive annual grasses under low and high nitrogen supply. - J. Arid Environ. 72: 1775-1784, 2008.

Kalaji M.H, Oukarroum A., Alexandrov V. et al.: Identification of nutrient deficiency in maize and tomato plants by in vivo chlorophyll $a$ fluorescence measurements. - Plant Physiol. Bioch. 81: 16-25, 2014.

Kalaji M.H., Nalborczyk E.: Gas exchange of barley seedlings growing under salinity stress. - Photosynthetica 25: 197-202, 1991.

Kattge J., Díaz S., Lavorel S. et al.: TRY - a global database of plant functional traits. - Glob. Change Biol. 17: 2905-2935, 2011.

Kryszak J., Domański P., Jokś W.: Use value of Festulolium braunii (K. Richter) A. Camus cultivars registered in Poland. Grassland Sci. Eur. 7: 436-437, 2002.

Leuschner C., Gebel S., Rose L.: Root trait responses of six temperate grassland species to intensive mowing and NPK fertilization: a field study in a temperate grassland. - Plant Soil 373: 687-698, 2013.

Mendiburu F.: Agricolae: Statistical Procedures for Agricultural Research. $R$ package version 1.1-3. Pp. 157. http://CRAN.Rproject.org/package=agricolae [accessed 0404 2016], 2012.

Nekrošas S., Kemešytė V.: Breeding of ryegrass and festulolium in Lithuania. - Zemdirbyste 94: 29-39, 2007.

Olszewska M.: Gas exchange parameters in Festulolium braunii (K. Richt.) A Camus grown in mixtures with legumes depending on multiple nitrogen rates. - Pol. J. Natur. Sci. 23: 48-72, 2008a.

Olszewska M.: Leaf greenness (SPAD) and yield of Festulolium braunii (K. Richt.) A. Camus grown in mixtures with legumes depending on multiple nitrogen rates. - Pol. J. Natur. Sc. 23 (2): 310-325, 2008b.

Ostonen I., Püttsepp Ü., Biel C. et al.: Specific root length as an indicator of environmental change. - Plant Biosyst. 141: 426442, 2007. 
Østrem L., Larsen A.: Winter survival, yield performance and forage quality of Festulolium cvs. for Norwegian farming. Grassland Sci. Eur. 13: 293-295, 2008.

Østrem L., Volden B., Larsen A.: Morphology, dry matter yield and phenological characters at different maturity stages of Festulolium compared with other grass species. - Acta Agr. Scand. B S. P. 63: 531-542, 2013.

Picon-Cochard C., Pilon R., Tarroux E. et al.: Effect of species, root branching order and season on the root traits of 13 perennial grass species. - Plant Soil 353: 47-57, 2012.

$R$ Development Core Team. $R$ : A Language and Environment for Statistical Computing. Pp. 3501. $R$ Foundation for Statistical Computing, Vienna 2013.

Ryser P.: The mysterious root length. - Plant Soil 286: 1-6, 2006.

Ryser P., Lambers H.: Root and leaf attributes accounting for the performance of fast- and slow-growing grasses at different nutrient supply. - Plant Soil 170: 251-265, 1995.

Shangguan Z.P., Shao M.A., Dyckmans J.: Nitrogen nutrition and water stress effects on leaf photosynthetic gas exchange and water use efficiency in winter wheat. - Environ. Exp. Bot.
44: 141-149, 2000.

Soussana J.F., Klumpp K., Ehrhardt F.: The role of grassland in mitigating climate change. - Grassland Sci. Eur. 19: 75-87, 2014.

Starck Z.: [Effect of stress conditions on coordination of photosynthetic production and resources allocation.] - Post. Nauk Rol. 1: 9-26, 2010. [In Polish]

Stypiński P., Mastalerczuk G.: Effect of management intensity on sward productivity and root mass of permanent meadow. Grassland Sci. Eur. 10: 441-444, 2005.

Svoboda P., Haberle J.: The effect of nitrogen fertilization on root distribution of winter wheat. - Plant Soil Environ. 52: 308-313, 2006.

Zhu Y., Fan X., Hou X. et al.: Effect of different levels of nitrogen deficiency on switchgrass seedling growth. - Crop J. 2: 223-234, 2014.

Živčák M., Olšovská K., Slamka P. et al.: Measurements of chlorophyll fluorescence in different leaf positions may detect nitrogen deficiency in wheat. - Zemdirbyste 101: 437-444, 2014. 